\section{Intravenous nicardipine for the treatment of renovascular hypertension due to fibromuscular dysplasia during pregnancy: A case study}

\author{
Toshitaka Mori, Kazushi Watanabe, Hiroshi Matsushita, Ai Fujimaki, \\ Chiharu Kimura, Atsumi Yoshida, Akihiko Wakatsuki
}

Reprint request to:

Toshitaka Mori, M.D., Ph.D., Department of Obstetrics and Gynecology, Aichi Medical University School of Medicine, Nagakute city, Aichi, 480-1195, Japan.

E-mail: mori1817@aichi-med-u. ac.jp

\section{Key words:}

fibromuscular dysplasia, pregnancy, renovascular hypertension

Received: March 4, 2014

Revised: May 7, 2014

Accepted: May 25, 2014

Department of Obstetrics and Gynecology, Aichi Medical University School of Medicine

Renovascular hypertension due to renal artery stenosis associated with fibromuscular dysplasia during pregnancy can lead to maternal and fetal complications. Although conventional percutaneous angiography and angioplasty are recommended for patients with renal artery stenosis due to fibromuscular dysplasia, the influence of these procedures on women and fetuses is unknown. A 27-year-old primigravida was diagnosed with renovascular hypertension possibly due to fibromuscular dysplasia. Percutaneous transluminal angioplasty in conjunction with renal angiography was initially planned, but she became pregnant and was referred to our department at 9 weeks of gestation. At 22 weeks of gestation, she was administered intravenous nicardipine (24-120 mg/day) for blood pressure control until 32 weeks of gestation. At 33 weeks 2 days of gestation, her hypertension was uncontrollable, and she delivered a healthy infant by cesarean section. Intravenous administration of nicardipine may present an effective and safe method to control blood pressure in women with fibromuscular dysplasia.

\section{Introduction}

Renovascular hypertension, a secondary hypertension caused by renal artery stenosis, accounts for $5 \%$ of all hypertension cases in the general population. However, it is uncommon during pregnancy, and published data are limited to a few case reports. ${ }^{1)}$ Fibromuscular dysplasia is a non-inflammatory, non-atherosclerotic vascular disease that involves small and medium-sized arteries, and contributes to the etiology of renal artery stenosis in childbearing women. ${ }^{2)}$ It is thus considered an important cause of hypertension during pregnancy. Percutaneous angiography and angioplasty are the gold standard for diagnosis and treatment of fibromuscular dysplasia. During pregnancy, however, patients are generally managed with medical therapy to avoid invasive diagnostic and therapeutic procedures. Pregnancy is terminated in some cases, given the risk of teratogenic effects from fetal radiation exposure. ${ }^{3)}$ On the other hand, long-term treatment with intravenous nicardipine has been reported to be an effective and safe method for treating hypertension during pregnancy. ${ }^{4}$
In our case, intravenous administration of nicardipine was effective and safe, leading to the successful delivery of a healthy infant at 33 weeks 2 days of gestation. Thus, intravenous nicardipine in the earlier stages of pregnancy in patients with fibromuscular dysplasia may prolong pregnancy and allow for fetal lung maturation.

\section{Case presentation}

A 27-year-old primigravida was admitted to the Department of Cardiovascular Medicine to examine severe hypertension in October 2012. She had a history of hypertension at age 22 and received treatment with antihypertensive medication. However, she did not continue the medication and was lost to follow-up. Doppler ultrasonography revealed bilaterally constricted renal arteries with elevated peak systolic velocities consistent with renal artery stenosis (Figure 1). She was diagnosed with renovascular hypertension possibly due to fibromuscular dysplasia. ECG, chest radiograph, and transthoracic echocardiography were normal. She had normal renal function with a blood urea nitrogen level 
T. Mori et al.
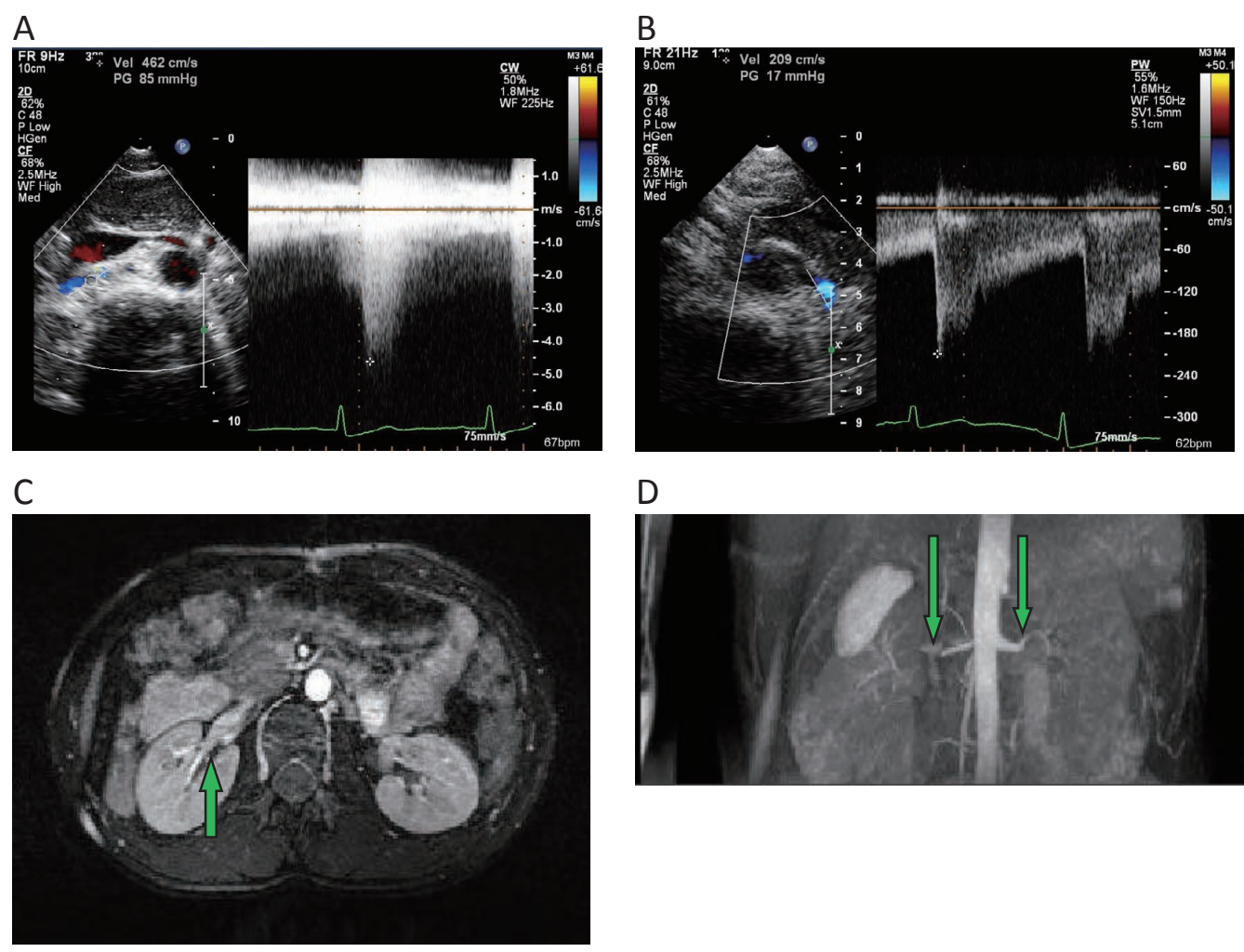

D

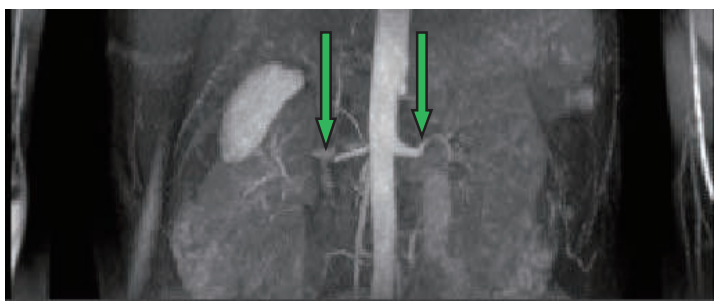

Figure 1. Ultrasound and MRI of the renal artery were examined 2 months before the pregnancy.

(A) Ultrasound of the right renal artery showing elevated peak systolic velocity of $462 \mathrm{~cm} / \mathrm{s}$.

(B) Ultrasound of the left renal artery showing elevated peak systolic velocity of $209 \mathrm{~cm} / \mathrm{s}$.

(C) MRI T2-weighted images shows narrowing in the distal site of right renal artery (arrow).

(D) MRI The distal sites of both renal arteries indicate stenosis (arrow).

of $9.5 \mathrm{mg} / \mathrm{dl}$ and serum creatinine concentration of 0.53 $\mathrm{mg} / \mathrm{dl}$. Plasma renin activity was elevated slightly, at $3.3 \mathrm{ng} / \mathrm{ml} / \mathrm{h}$. Percutaneous transluminal angioplasty in conjunction with renal angiography was initially planned, but as she became pregnant, she was referred to our department at 9 weeks of gestation to manage pregnancy under conditions of chronic hypertension. A physical examination revealed a blood pressure of $196 / 120 \mathrm{mmHg}$. We gave her a thorough explanation regarding the need of further examination and treatment for hypertension, as well as the possibility of miscarriage. She and her family hoped to continue pregnancy without undergoing angiography and percutaneous transluminal angioplasty given the potential teratogenic effects due to fetal radiation exposure. Therefore, she was started on $750 \mathrm{mg}$ of methyldopa three times daily. Her blood pressure was controlled, transiently going below 150/90 $\mathrm{mmHg}$. At 22 weeks of gestation, however, she required hospitalization due to poorly controlled blood pressure $(160 / 100 \mathrm{mmHg})$. She was started on intravenous nicardipine $(24-120 \mathrm{mg} /$ day) during hospitalization. Blood pressure was adequately controlled for a while, with systolic and diastolic blood pressures in the range of $140-150 \mathrm{mmHg}$ and $80-90 \mathrm{mmHg}$, respectively, without any severe maternal or fetal adverse effects. Weekly ultrasound scans and a daily non-stress test were performed to evaluate the well-being of the fetus. The decrease in blood pressure did not cause adverse effects such as fetal growth restriction or fetal distress. However, her hypertension could not be controlled any more at 32 weeks of gestation; she had headaches, and despite intravenous nicardipine, her systolic and diastolic blood pressures were $160-170 \mathrm{mmHg}$ and $90-100 \mathrm{mmHg}$, respectively. Thus, a decision was made to perform emergency cesarean section at 33 weeks 2 days of gestation. She delivered a healthy female infant weighing $1,840 \mathrm{~g}$. The subsequent neonatal course was uneventful. After delivery, her blood pressure improved remarkably, averaging 130/80 $\mathrm{mmHg}$, on $750 \mathrm{mg}$ of methyldopa three times daily. She was discharged without severe complications (Figure 2). 


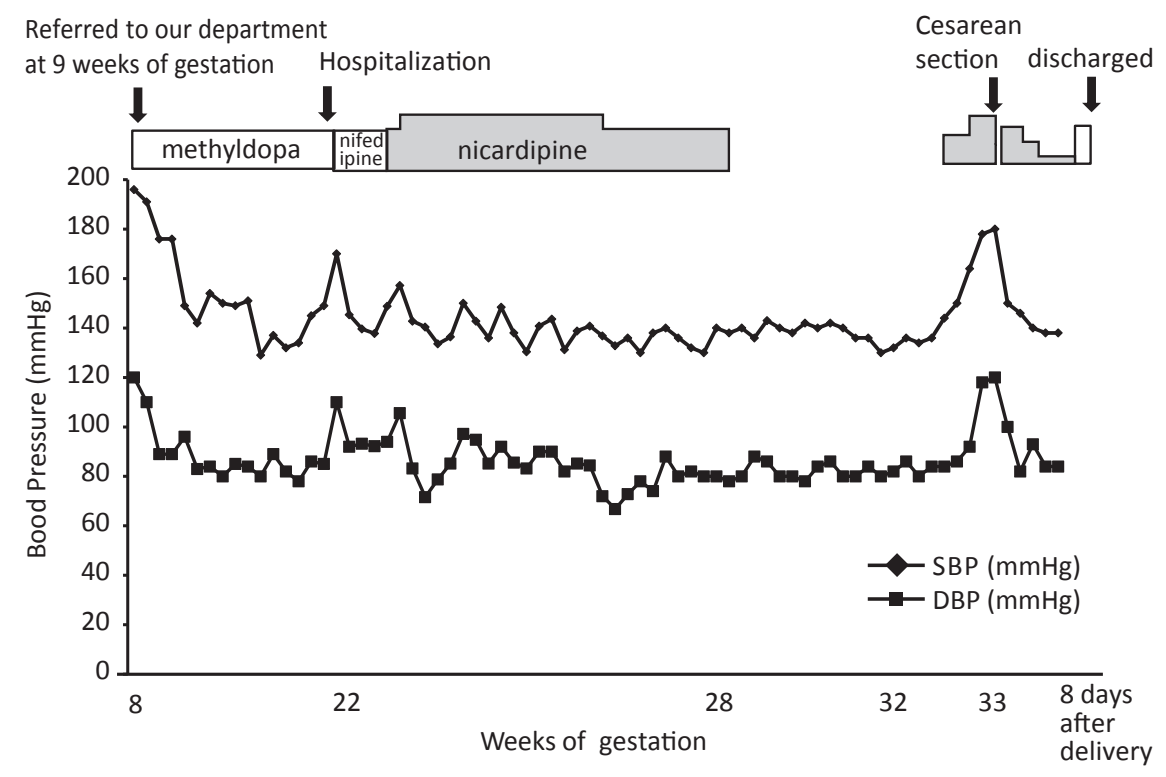

Figure 2. Clinical course of the patient.

The clinical course including the period before hospitalization until the time of discharge after delivery is depicted. Blood pressure and antihypertensive agents are shown.

\section{Discussion}

Fibromuscular dysplasia, a non-atherosclerotic angiopathy of unknown etiology, ${ }^{2}$ often affects women between ages of 15 and 50 years, but is rare during pregnancy. It commonly affects renal and carotid arteries and has also been observed in almost every artery in the body. ${ }^{5,6}$ Patients with renovascular hypertension due to fibromuscular dysplasia might be at high risk of developing severe preeclampsia. Thorsteinsdottir et al. ${ }^{7)}$ reported on a series of patients with poor pregnancy outcomes due to severe preeclampsia and placental abruption at gestational week 20. In patients with renal artery stenosis, successful pregnancies have been reported 1 year after revascularization. They also reviewed previous reports regarding two patients who experienced second-trimester pregnancy losses secondary to intrauterine fetal death, and one patient who electively terminated pregnancy due to possible teratogenic fetal effects after radiation exposure. ${ }^{3)}$

Percutaneous angiography and angioplasty are generally useful for diagnosis and treatment of fibromuscular dysplasia. Cohen et al. ${ }^{8)}$ reported on a 18 -week pregnant woman with fibromuscular dysplasia, who underwent angioplasty for both sides of renal arteries guided by ultrasound findings and remained normotensive without medications through the rest of her pregnancy. According to American College of Obstetricians and Gynecologists guidelines for diagnostic imaging during pregnancy, the probability of harmful effects on fetuses is small if radiation exposure is limited to less than $5 \mathrm{rad}$ (i.e., $50 \mathrm{mGy}){ }^{3)}$ Although angiography and percutaneous transluminal angioplasty are common for diagnosis and treatment of renal artery stenosis, patients are generally managed with medical therapy during pregnancy to avoid invasive diagnostic and therapeutic procedures. Moreover, termination of pregnancy is sometimes decided given the risk of teratogenic effects due to fetal radiation exposure. Our patient required further diagnostic studies for her renal artery stenosis before she became pregnant, and angiography and percutaneous transluminal angioplasty were initially planned. However, we gave up on further invasive examination during the pregnancy in accordance with patient and family wishes.

The need for medications even after angioplasty has been suggested. Heyborne et al. ${ }^{1)}$ reported a case of a woman with high grade stenosis in the right artery, whose blood pressure was resistant to oral antihypertensive agents and underwent transluminal angioplasty at 26 weeks of gestation. Although her blood pressure was controlled to some extent, she required medication even after angioplasty until delivery by cesarean section at 29 weeks of gestation due to intrauterine fetal growth restriction and absent end diastolic velocity in the umbilical artery.

Intravenous antihypertensive agents are generally superior to oral medications in controlling blood pressure. Seki et al. ${ }^{4}$ reported that long-term treatment with nicardipine is effective and safe in pregnant patients with hypertension. They divided fifty preeclamptic women 
into three groups according to the length of intravenous nicardipine treatment, and concluded that long-term treatment is as effective and safe as short- and mediumterm treatments. In another study, Suzuki et al. ${ }^{9)}$ reported that in women with uncontrollable severe hypertension, intravenous antihypertensive medication may be needed to lower blood pressure to the desired goal. They also recommend the use of a sliding scale in pregnant women. However, no report has described long-term treatment with intravenous nicardipine (in place of oral medication) in women with fibromuscular dysplasia during pregnancy. In our case, intravenous administration of nicardipine led to prolongation of the duration of gestation, and the patient was able to deliver a healthy infant at 33 weeks of gestation. Intravenous nicardipine in earlier stages of pregnancy in patients with fibromuscular dysplasia might afford a better outcome by allowing for fetal lung maturation without severe complications to the mother and fetus.

Intravenous nicardipine in earlier stages of pregnancy may be considered a safe and effective treatment option in women with renovascular hypertension before introduction of angioplasty, even if oral medications are ineffective.

\section{Acknowledgements}

This study received no foundational support.

\section{Conflict of interest}

None.

\section{References}

1. Heyborne KD, Schultz MF, Goodlin RC, Durham JD. Renal artery stenosis during pregnancy: a review. Obstet Gynecol Surv. 1991; 46: $509-514$.

2. Olin JW. Recognizing and managing fibromuscular dysplasia. Cleve Clin J Med. 2007; 74: 273-274, 277-282.

3. ACOG Committee on Obstetric Practice. Guidelines for diagnostic imaging during pregnancy. Obstet Gynecol. 2004; 104: 647-651.

4. Seki H. Takeda S. Kinoshita K. Long-term treatment with nicardipine for severe pre-eclampsia. Int J Gynaecol Obstet. 2002; 76: $135-141$.

5. Olin JW, Sealove BA. Diagnosis, management, and future developments of fibromuscular dysplasia. J Vasc Surg. 2011; 53: 826-836.e1.

6. Dawley B, Ritchie A. Carotid and vertebral arterial fibromuscular dysplasia masquerading as severe preeclampsia: a case report. W V Med J. 2011; 107: 12-14.

7. Thorsteinsdottir B, Kane GC, Hogan MJ, Watson WJ, Grande JP,Garovic VD. Adverse outcomes of renovascular hypertension during pregnancy. Nat Clin Pract Nephrol. 2006; 2: 651-656.

8. Cohen DL, Townsend RR, Clark TW. Renal artery stenosis due to fibromuscular dysplasia in an 18-week pregnant woman. Obstet Gynecol. 2005; 105: 1232-1235.

9. Suzuki Y, Adachi T, Ohno Y et al. Committee report on cerebrovascular disorders, including eclampsia and emergency medical services, of the Japan Society for the Study of Hypertension in Pregnancy. Hypertens Res Pregnancy. 2013; 1: 8-12. 